\title{
Être intervenant auprès de parents ayant un trouble de personnalité limite en protection de la jeunesse : quand la souffrance de l'un se conjugue au désarroi de l'autre Working with parents with borderline personality disorder in youth protection services: When suffering is combined with dismay
}

\author{
Lise Laporte, Louise Baillargeon, Isabelle Sanchez et Lyne Desrosiers
}

Volume 43, numéro 2, 2014

URI : https://id.erudit.org/iderudit/1061187ar

DOI : https://doi.org/10.7202/1061187ar

Aller au sommaire du numéro

Éditeur(s)

Revue de Psychoéducation

ISSN

1713-1782 (imprimé)

2371-6053 (numérique)

Découvrir la revue

Citer cet article

Laporte, L., Baillargeon, L., Sanchez, I. \& Desrosiers, L. (2014). Être intervenant auprès de parents ayant un trouble de personnalité limite en protection de la jeunesse : quand la souffrance de l'un se conjugue au désarroi de l'autre. Revue de psychoéducation, 43(2), 323-347. https://doi.org/10.7202/1061187ar

\section{Résumé de l'article}

Environ 22,5\% des mères d'enfants pris en charge par les services de protection de la jeunesse présente un trouble de personnalité limite avéré ou probable. Il existe cependant très peu d'informations sur les défis que doivent relever les intervenants psychosociaux qui travaillent avec cette clientèle dans le contexte d'un mandat de protection. Cette étude qualitative s'est penchée sur cette problématique particulière. Les données ont été recueillies selon la méthode du groupe cible (focus group) auprès de 104 intervenants du Centre jeunesse de Montréal-Institut universitaire dans le cadre de six rencontres. Les résultats suggèrent que ceux-ci perçoivent que leurs interventions auprès de ces familles sont soumises à un ensemble de contingences qui rendent leur travail particulièrement complexe et exigeant. Trois thèmes principaux reliés aux préoccupations et aux difficultés à l'égard de leur pratique et de leurs besoins ont ainsi été mis en évidence. Le premier concerne les difficultés d'intervention liées au contexte interpersonnel. Les analyses révèlent notamment que devoir concilier protection des enfants et aide aux parents ou encore parler aux enfants du trouble mental de leur parent s'avèrent difficile pour eux. Le second thème, les enjeux liés au contexte environnemental, concernent leurs perceptions des contingences associées à l'organisation interne des services en centre jeunesse et à la collaboration avec les partenaires externes (milieu judiciaire et psychiatrie adulte). L'impact des contextes interpersonnel et environnemental sur les intervenants eux-mêmes constitue le troisième thème. Les analyses suggèrent que la prise en charge des familles touchées par ce trouble mental sollicite énormément les intervenants sur le plan affectif et cognitif et leur pose des défis cliniques quotidiens. Enfin, cet article souligne l'importance de se préoccuper des défis auxquels font face les intervenants oeuvrant auprès de familles touchées par un trouble de personnalité limite et présente quelques recommandations d'ordre organisationnel et clinique pour mieux les soutenir.
Tous droits réservés (C) La Corporation de la Revue Canadienne de Psycho-Éducation, 2014
Ce document est protégé par la loi sur le droit d'auteur. L’utilisation des services d’Érudit (y compris la reproduction) est assujettie à sa politique d'utilisation que vous pouvez consulter en ligne.

https://apropos.erudit.org/fr/usagers/politique-dutilisation/ 


\title{
Être intervenant auprès de parents ayant un trouble de personnalité limite en protection de la jeunesse : quand la souffrance de l'un se conjugue au désarroi de l'autre.
}

\author{
Working with parents with borderline personality disorder in \\ youth protection services: When suffering is combined with \\ dismay.
}

L. Laporte ${ }^{1}$

L. Baillargeon ${ }^{2}$

I. Sanchez ${ }^{3}$

L. Desrosiers ${ }^{4}$

1 Centre de recherche, Centre jeunesse de Montréal-Institut universitaire.

Programme des troubles de la personnalité, Centre universitaire de santé McGill.

2 Centre jeunesse de MontréalInstitut universitaire.

3 Université du Québec à Montréal

4 Centre de recherche, Centre jeunesse de Montréal-Institut universitaire.

Département d'ergothérapie, Université du Québec à

Trois-Rivières.

Cet article a été rendu possible grâce à une subvention du GRAVE-Ardec et du CJM-IU.

Les auteurs n'ont aucun conflit d'intérêt.

\section{Correspondance :}

Lise Laporte

Centre de recherche

Centre jeunesse de Montréal-

Institut universitaire (CJM-IU)

1001, boul. de Maisonneuve Est

$7^{\mathrm{e}}$ étage

Montréal (Québec)

CANADA H2L 4R5

Tél. : 514-896-3470

Télécopieur : 514-896-3400

lise.laporte@cjm-iu.qc.ca

\section{Résumé}

Environ 22,5\% des mères d'enfants pris en charge par les services de protection de la jeunesse présente un trouble de personnalité limite avéré ou probable. II existe cependant très peu d'informations sur les défis que doivent relever les intervenants psychosociaux qui travaillent avec cette clientèle dans le contexte d'un mandat de protection. Cette étude qualitative s'est penchée sur cette problématique particulière. Les données ont été recueillies selon la méthode du groupe cible (focus group) auprès de 104 intervenants du Centre jeunesse de Montréal-Institut universitaire dans le cadre de six rencontres. Les résultats suggèrent que ceux-ci perçoivent que leurs interventions auprès de ces familles sont soumises à un ensemble de contingences qui rendent leur travail particulièrement complexe et exigeant. Trois thèmes principaux reliés aux préoccupations et aux difficultés à l'égard de leur pratique et de leurs besoins ont ainsi été mis en évidence. Le premier concerne les difficultés d'intervention liées au contexte interpersonnel. Les analyses révèlent notamment que devoir concilier protection des enfants et aide aux parents ou encore parler aux enfants du trouble mental de leur parent s'avèrent difficile pour eux. Le second thème, les enjeux liés au contexte environnemental, concernent leurs perceptions des contingences associées à l'organisation interne des services en centre jeunesse et à la collaboration avec les partenaires externes (milieu judiciaire et psychiatrie adulte). L'impact des contextes interpersonnel et environnemental sur les intervenants eux-mêmes constitue le troisième thème. Les analyses suggèrent que la prise en charge des familles touchées par ce trouble mental sollicite énormément les intervenants sur le plan affectif et cognitif et leur pose des défis cliniques quotidiens. Enfin, cet article souligne l'importance de se préoccuper des défis auxquels font face les intervenants œuvrant auprès de familles touchées par un trouble 
de personnalité limite et présente quelques recommandations d'ordre organisationnel et clinique pour mieux les soutenir.

Mots-clés: parents; trouble de personnalité limite; intervention psychosociale; services de protection de la jeunesse.

\begin{abstract}
About $22.5 \%$ of mothers of children followed by Youth Protection Services have a known or probable borderline personality disorder. There are however very little information on the challenges faced by psychosocial workers who work with this clientele in the context of a protection mandate. This qualitative study focused on this particular issue. Data was collected during six focus groups in which 104 case workers from Centre jeunesse de Montréal-Institut universitaire participated. The results suggest that they perceive their interventions with these families to be subject to a set of contingencies that make their work particularly complex and demanding. Three main themes in relation to their concerns and challenges regarding their practice and in relation to their needs were highlighted. The first theme regards intervention difficulties related to the interpersonal context. The analyses show for example that trying to reconcile child protection and support for parents, or to talk to the children about their parents' mental disorder prove to be particularly difficult. The second theme related to the context of their working environment concerns their perceptions of the contingencies associated with the internal organization of services and with the collaboration with partners (judiciary milieu and adult psychiatry). The impact of the interpersonal and environmental contexts on the caseworkers constitutes the third theme. Participants suggested that the management of families impacted by this mental disorder puts an important strain on them, both emotionally and cognitively. Finally, this article highlights the importance of addressing the daily challenges faced by those working with families affected by borderline personality disorder and presents some organizational and clinical recommendations in order to better support the workers.
\end{abstract}

Keywords: parents, borderline personality disorder, psychosocial intervention, youth protection services.

Au Québec, les intervenants qui travaillent en protection de la jeunesse sont confrontés quotidiennement à des familles aux prises avec une multitude de problèmes psychosociaux. Parmi ceux-ci, les troubles mentaux chez les parents sont particulièrement préoccupants et prévalents. En effet, une étude menée récemment dans deux centres de protection de la jeunesse (Laporte \& Friedland, 2013) rapporte que $61 \%$ des mères d'enfants pris en charge par la protection de la jeunesse souffriraient de troubles mentaux. De ce groupe, plus du tiers présenteraient un trouble de personnalité limite (TPL) avéré ou probable. On peut donc estimer qu'un nombre important de mères, soit environ $22,5 \%$, sont aux prises avec ce trouble mental sévère.

Il existe très peu d'informations dans la littérature sur les défis que doivent relever les intervenants psychosociaux qui travaillent avec ces parents dans le contexte de la protection de la jeunesse. Cette étude exploratoire propose de rendre compte de leurs constatations, préoccupations et difficultés à l'égard de leur pratique ainsi que de leurs besoins pour intervenir avec cette clientèle particulière. 
Le trouble de personnalité limite est un trouble mental sérieux et complexe. II est caractérisé par des relations interpersonnelles intenses et chaotiques, une identité diffuse, une hypersensibilité au rejet et à l'abandon et des difficultés importantes de régulation émotionnelle (American Psychiatric Association, 2013). Les personnes qui en souffrent présentent des comportements impulsifs allant de l'automutilation, aux abus de substances, aux menaces et aux tentatives de suicide, qui, dans $10 \%$ des cas, seront fatales (Paris \& Zweig-Frank, 2001). Ce trouble de santé mentale est associé à un niveau élevé de souffrance et de détresse psychologique et entraîne des effets dévastateurs pour la personne et pour sa famille. Les études qui se sont penchées sur les enfants de parents ayant un TPL rapportent une prévalence élevée de psychopathologie chez ces derniers (Barlow, Spitzer, Grabe, Kessler, \& Freyberger, 2006; Coulborn-Faller \& Bellamy, 2000). D'après Rutter et Quinton (1984), vivre avec un parent qui présente ce trouble sévère de la personnalité est le facteur qui prédit le mieux le développement d'un problème de santé mentale. Lorsque ces enfants sont en plus victimes d'abus ou de négligence, ils risquent aussi de développer à leur tour un trouble sévère de la personnalité (Johnson et al., 2000).

Les écrits sur le trouble de personnalité limite font état des grandes difficultés que connaissent les thérapeutes avec cette clientèle (Paris, 1996). En effet, les personnes ayant un TPL sont reconnues pour susciter des réactions intenses de contre-transfert (Betan, Heim, Conklin, \& Westen, 2005; Bouchard, 2010; Cousineau, 1996; Normandin \& Ensink, 2007), lesquelles peuvent interférer sérieusement avec l'intervention et peuvent même parfois lui nuire (Dawson, 1988; Denis, 1990; Fonagy \& Bateman, 2006; Lafleur, St-Onge, Rouleau, Tremblay, \& Villeneuve, 2004). Le développement et le maintien de la relation d'aide auprès de ces personnes représente donc un défi clinique important (Bergeron-Leclerc, 2010). Les professionnels de la santé mentale considèrent que les personnes souffrant d'un TPL sont les plus ardues à traiter (Cleary, Siegfried, \& Walter, 2002; Cousineau, 1996), notamment parce qu'elles les soumettent à une charge émotionnelle importante (Bessette, 2010). Ce diagnostic est également considéré comme le plus complexe à comprendre (Fruzzetti, Shenk, \& Hoffman, 2005; Paris, 2009).

Par ailleurs, bien qu'il existe plusieurs traitement dont l'efficacité a été démontrée par des données probantes (Bateman \& Fonagy, 2000; Bateman \& Fonagy, 2009; Biskin \& Paris, 2012; Linehan et al., 2006), les interventions développées jusqu'à maintenant ne sont pas nécessairement appropriées pour une clientèle de parents et particulièrement de parents maltraitants (Marziali, Damianakis, \& Trocmé, 2003). En effet, les atteintes spécifiques en regard des habiletés parentales n'ont pas été véritablement considérées par les différentes approches thérapeutiques reconnues pour le TPL.

La Loi sur la protection de la jeunesse (LPJ) du Québec favorise la mobilisation rapide des parents afin qu'ils participent activement à la résolution de leurs difficultés familiales et rectifient la situation de compromission de leur enfant (LeBlanc, 2010). Le mandat des intervenants d'accompagner le parent dans cette démarche se complexifie lorsque celui-ci présente un TPL. En effet, l'intervention constitue un défi plus important puisque la motivation à changer fluctue dans le 
temps et varie considérablement chez les personnes atteintes de ce trouble (Bouchard, 2010). De plus, la sensibilité au rejet, les difficultés relationnelles et l'intensité émotionnelle propre à ce trouble mental peuvent exacerber leurs réactions défensives et hostiles vis-à-vis la nécessité d'effectuer des changements au niveau de leurs attitudes parentales. Éprouvant davantage de difficultés à s'ajuster que les autres parents, leurs enfants risquent d'être confiés plus rapidement à un milieu de vie stable et sécurisant à l'extérieur de la famille.

II existe très peu de données précises sur les attitudes adoptées par les intervenants psychosociaux envers les parents ayant un TPL dans le contexte d'un mandat de protection. Cependant, il est reconnu que les personnes présentant un TPL constituent le groupe le plus stigmatisé, celui subissant le plus de préjugés, de rejets et de jugements négatifs de la part des institutions, de la population générale et même des cliniciens qui tendent à éprouver moins d'empathie pour cette clientèle (Aviram, Brodsky, \& Stanley, 2006). Ces perceptions font en sorte que les personnes présentant un TPL sont fréquemment discréditées et blâmées pour leurs difficultés (Crawford et al., 2007) et conséquemment privées de l'aide dont elles auraient besoin. Les intervenants sociaux rencontrés par Stanley et ses collègues (2003) rapportent à cet égard qu'ils attribuaient trop souvent l'étiquette de TPL aux parents difficiles, laquelle servait notamment à justifier de ne pas offrir les services normalement requis. De plus, ces intervenants se montraient très pessimistes quant à leurs capacités parentales ce qui les amenait à procéder à des placements plus hâtifs (Stanley \& Manthorpe, 2001).

Des intervenants du champ social en France se questionnent également sur leur rôle professionnel et leur efficacité personnelle. Confrontés aux problèmes de santé mentale de leur clientèle, à la complexité des situations et aux conditions difficiles de leur pratique, ils admettent avoir le sentiment de ne pas pouvoir faire correctement leur métier et rapportent aussi l'impression de ne plus répondre adéquatement aux situations auxquelles ils se heurtent (Ravon, Decrop, Ion, Laval, \& Vidal-Naquet, 2008; St-Jacques, Lessard, Beaudoin, \& Drapeau, 2000). Un rapport du Ministère français de la santé souligne que la détresse des intervenants face à ces parents ne doit pas être imputée à leur incompétence, mais bien au besoin de développement de leurs pratiques (Ministère de la Santé et des Solidarités, 2008).

Développer le savoir-faire, améliorer le sentiment d'efficacité et prévenir l'épuisement professionnel chez les intervenants qui travaillent avec une clientèle présentant un TPL constituent donc des enjeux bien réels de la pratique en protection de la jeunesse. Cependant, il apparaît d'abord nécessaire de cerner les réalités et les enjeux propres à la pratique en centre jeunesse au Québec.

Cette étude exploratoire a donc pour objectif de cerner les défis et les besoins des intervenants œuvrant dans le milieu de la protection de la jeunesse auprès des parents ayant un TPL avéré ou dont les caractéristiques sont perçues par les intervenants comme des traits de personnalité limite. Elle propose de mettre en lumière leurs observations et leurs représentations à l'égard du travail auprès de familles touchées par le TPL afin d'en dégager des pistes de solution pour bonifier leurs interventions. 


\section{Échantillon}

\section{Méthode}

Les données ont été recueillies selon la méthode du groupe cible (focus group). Le groupe a été utilisé comme une entité (Baribeau, 2010) et les propos rapportés constituent ce qui est vécu et rapporté par les membres des groupes rencontrés. Six groupes cibles réunissant des intervenants du service de protection de la jeunesse du Centre jeunesse de Montréal-Institut universitaire (CJM-IU) ont été constitués. Ces groupes ont été formés à partir d'équipes de travail existantes. Ainsi, au total, cent quatre (104) intervenantes et intervenants ont participé à un groupe cible.

Les équipes qui ont servi à constituer les groupes ont été sélectionnées et recrutées en fonction de leur intérêt à participer et selon les différents secteurs d'intervention (services à l'enfance et à l'adolescence, les services offerts en territoire et à l'interne) afin de diversifier l'échantillon. Quatre-vingt-dix pourcent des membres de ces équipes, qui sont composées majoritairement de femmes, œuvrent directement auprès des jeunes (travailleurs sociaux, éducateurs et psycho éducateurs). Les autres participants proviennent de divers services et ont comme mandat de soutenir les intervenants dans leur travail quotidien (chefs de service, infirmiers, psychologues). Dans le cadre de la présente recherche, les intervenants de cinq équipes étaient rattachés à l'application des mesures, c'est-à-dire au secteur du CJM-IU qui a la responsabilité de mettre en place les services dont la famille a besoin et d'en assurer le suivi. Tous les membres de ces équipes étaient invités à participer à la discussion et tous ceux qui étaient présents à la réunion ont accepté d'y participer sur une base volontaire. Un sixième groupe provenant du secteur de la réadaptation à l'interne était composé de divers intervenants intéressés par la question à l'étude.

\section{Déroulement des rencontres}

Bien que l'objet de la rencontre ait été présenté aux intervenants comme une étude sur le travail auprès de parents ayant un trouble de santé mentale, les entretiens n'ont porté que sur les parents ayant un TPL, le trouble de santé mentale qui, selon les intervenants, pose le plus de difficultés dans leur pratique et les met le plus souvent en échec. Les entretiens de groupe d'une durée moyenne de 2 heures ont été menés par deux des auteures de l'article, toutes deux ayant l'expérience de diriger des groupes de discussion. Les participants réagissaient aux questions ouvertes posées par les animatrices et faisaient part de leurs constatations, préoccupations et difficultés à l'égard de leur pratique auprès des parents ayant un TPL. Étant donné que les parents en centre jeunesse n'ont pas nécessairement de diagnostic formel de TPL, l'entrevue de recherche portait autant sur le travail des intervenants auprès de parents présentant des caractéristiques d'un TPL (un TPL soupçonné par les intervenants) que sur le travail auprès de parents qui avaient un TPL avéré avec diagnostic au dossier. 
Toutes les discussions ont été enregistrées avec le consentement des participants (Palmer, Larkin, De Visser, \& Fadden, 2010). Ces entretiens ont été transcrits et transférés sur le logiciel d'analyse NVivo version 7 pour être traités selon une méthode d'analyse thématique (Mayer \& Deslauriers, 2000). Des analyses de contenu des réponses données par l'ensemble des participants ont ensuite été réalisées. La méthode d'analyse thématique utilisée comporte les étapes suivantes : lecture de toutes les entrevues et choix des unités informationnelles à conserver, définition des catégories et classification des unités de sens, analyse de contenu des catégories et interprétation des résultats.Cette étude s'inscrit donc dans une perspective exploratoire servant à mieux comprendre les « perceptions, les sentiments, attitudes et motivations » (Touré, 2010, p. 11) ainsi que les besoins des intervenants dans leur travail auprès de parents ayant un TPL avéré ou probable.

\section{Résultats}

L'analyse des propos des intervenants travaillant à l'enfance et à l'adolescence et au sein de différents services a permis de mettre en lumière trois thèmes principaux : 1) les difficultés d'intervention liées au contexte interpersonnel (les difficultés à l'égard des parents et les préoccupations à l'égard des enfants); 2) les difficultés d'intervention liées au contexte environnemental (l'organisation interne et les collaborations externes) et 3) l'impact des contextes interpersonnel et environnemental sur les intervenants eux-mêmes. Ces derniers ont également exprimé leurs besoins dans une optique d'amélioration des pratiques. Ces préoccupations ont été intégrées à la présentation des trois thèmes principaux.

L'ordre de présentation des thèmes n'est pas lié à leur importance ni à leur poids relatif. Les citations présentées sont des extraits intégraux de verbatim. Elles ont été choisies en fonction de leur concision et de leur justesse pour illustrer chacun des thèmes.

\section{Les difficultés d'intervention liées au contexte interpersonnel}

Les difficultés d'intervention à l'égard des parents. Lors des entrevues, la grande majorité des intervenants mentionnent être fortement préoccupés par la complexité de l'intervention auprès des parents qui semblent présenter un TPL. Ils évoquent leur manque de connaissance sur la maladie mentale en général, la difficile évaluation de la nature exacte du trouble du parent ou encore le manque de clarté dans leur mandat, autant d'éléments qui, selon leurs dires, auraient un impact sur leur intervention.

Le manque de connaissance en santé mentale. Plusieurs intervenants rapportent avoir eu une formation minimale en santé mentale, lors de leurs études en travail social ou en psychoéducation, et en cours d'emploi. Ils disent ne pas se sentir préparés à intervenir au quotidien et à composer avec les résistances, les crises et les difficultés particulières des familles au sein desquelles un parent présente un TPL. Ils soulignent la complexité d'être en relation avec ces parents, ne 
sachant comment gérer leur imprévisibilité, leur explosivité, leurs attaques et leurs humeurs changeantes.

Être capable de voir que c'est un TPL, c'est une chose et c'est déjà bon. Mais comment faire après? Quand le parent est en état de crise psychotique, c'est le néant.

Il semble également que lorsqu'un parent est sous médication, le manque de connaissances sur la pharmacothérapie et les enjeux liés à la prise de médicaments soulèveraient de nombreuses questions : doivent-ils demander aux parents s'ils prennent des médicaments? Que fait-on quand le parent en prend trop ou pas assez, arrête brutalement d'en prendre ou en donne à son enfant? Quels sont les effets des médicaments sur la capacité du parent à s'occuper de son enfant?

Généralement, les intervenants moins expérimentés souhaitent acquérir des connaissances de base sur le TPL. Tous, par contre, aimeraient qu'une formation continue adaptée aux besoins et à la réalité des intervenants en protection de la jeunesse soit mise en place afin d'offrir des balises quant à l'accompagnement au quotidien, et ce tant auprès des parents qu'auprès des adolescents susceptibles de développer ce problème.

L'incertitude reliée au diagnostic. Selon les dires des intervenants, l'incertitude quant à la nature et la sévérité du trouble de la personnalité du parent lorsqu'il n'y a pas de diagnostic au dossier soulève une autre grande difficulté. Ils soulignent les risques de juger eux-mêmes de la situation et de conclure à un problème clinique qui ne serait pas nécessairement valide.

Ainsi, de l'avis de certains intervenants, un parent difficile risque de recevoir rapidement une étiquette de TPL. Par ailleurs, d'autres participants notent qu'il faut parfois beaucoup de temps avant de se rendre compte que le parent souffre de ce trouble mental. Les renseignements sur la santé mentale des parents ne seraient pas inscrits de façon rigoureuse et facilement repérable dans les dossiers. De plus, cette situation est d'autant problématique que le système informatique administratif ne consigne pas la présence d'un trouble mental chez les parents. En effet, même lorsqu'un problème de santé mentale chez le parent est à l'origine du signalement, le diagnostic n'est jamais inscrit. Les intervenants souhaitent que les informations sur la santé mentale soient mieux gérées, plus complètes, et que la procédure d'accès au dossier soit moins lourde.

Quand on a des parents qui ont des stress énormes, de l'anxiété, s'il n'y a pas de diagnostic dans le dossier, c'est difficile de faire la part des choses. À les voir aller une fois par mois, on ne peut pas tirer des conclusions sur leur santé mentale. Si à chaque fois qu'on appelle la mère, elle est sur les nerfs...Est-ce que c'est juste le fait que c'est une madame qui est mal à l'aise, qui n'a pas d'habiletés sociales, et qui est bête avec nous qu'on va tirer des conclusions? C'est peut-être correct de dire qu'elle a une santé mentale fragile, 
mais de là à identifier un trouble de la personnalité clair, faut faire attention.

Lorsqu'il y a un diagnostic au dossier, les intervenants considéraient qu'il est plus facile de distinguer ce qui est de l'ordre des symptômes du TPL dans le comportement des parents.

Ces gens-là amènent à ce qu'on soit mêlé. On est là pour les aider, mais pour eux, on est le problème! Quand il y a un diagnostic, on est plus capable de se mettre à l'extérieur de la situation.

Par ailleurs, les intervenants sont bien conscients que connaître le diagnostic ne résoudrait pas toutes leurs difficultés. Par exemple, lorsque le parent a un diagnostic formel de TPL, ce dernier s'en sert parfois pour se déresponsabiliser. D'autres parents au contraire nient qu'ils ont un problème, ou refusent le diagnostic. Certains manquent d'introspection concernant leur problème et son impact sur leur capacité de prendre soin de leur enfant. Ces répondants disent consacrer beaucoup de temps et d'énergie à essayer d'amener les parents à reconnaître leurs symptômes et à consulter. Du point de vue des intervenants, avec ou sans diagnostic, ils se retrouvent sans moyen pour travailler avec ces parents.

Ce que je trouve difficile surtout c'est quand les gens ne l'acceptent pas [le diagnostic], qu'ils refusent d'en parler. Là, ça devient difficile parce que nous, on est dans l'obligation de poursuivre le travail avec eux (...) on a la responsabilité de ce qui est précieux pour eux, leurs enfants. Ce sont les cas que je trouve les plus difficiles.

Les intervenants soulignent que l'absence du diagnostic du parent au dossier est encore plus problématique lorsque le jeune n'est signalé qu'à l'adolescence. D'après eux, toute l'attention serait mise sur les comportements du jeune (vol, fugue), ce qui a pour conséquence d'occulter que des problèmes importants chez le parent peuvent aussi avoir un impact sur les comportements problématiques de l'adolescent

La protection des enfants ou l'aide aux parents. Bien que tous conçoivent que la protection des enfants soit leur principal mandat, des intervenants rapportent qu'ils considèrent parfois difficile de concilier cet aspect et celui de l'aide au parent souffrant d'un TPL. Plusieurs intervenants perçoivent cela comme un double mandat dans lequel ils se sentent coincés. Ils déplorent devoir remplacer la relation d'aide à l'enfant par une intervention auprès du parent, qu'ils décrivent généralement faite en urgence, sous pression et parfois sans trop de recul : « le client c'est l'enfant, mais on est pris à gérer les crises du parent ».

Je me souviens d'une famille de 3 enfants. L'intervention avec le parent demandait un temps exagéré et au bout de 3 mois, aucun suivi n'avait été fait auprès des enfants. L'intervenante avait été happée par le parent; le drainage des énergies avait causé l'abandon de l'intervention auprès de l'enfant. 
À l'inverse, d'autres intervenants estiment que leur mandat est plus clair : il ne s'agirait pas d'aider les parents, mais d'assurer la protection et le développement de leurs enfants. Bien qu'ils rapportent encadrer et outiller les parents, ils soulignent que si l'enfant ne progresse pas, l'aide au parent est délaissée, car ils considèrent devoir toujours travailler dans l'intérêt de l'enfant. Ces intervenants déplorent tout de même que rien ne soit mis en place pour les parents.

On est ici pour assurer la mise en place de services à la disposition du parent pour augmenter ses compétences parentales. On a un mandat de contrôle social, pas de relation d'aide. Ici [au centre jeunesse], aucune thérapie ne se donne, il n'y a aucun spécialiste pour cela. La priorité est la protection de l'enfant. Mais je trouve que quand un parent est en difficulté dans son rôle de parent, d'individu et de citoyen, on ne fait rien.

Les préoccupations des intervenants à l'égard des enfants. L'analyse du discours des intervenants a fait ressortir deux préoccupations reliées aux enfants des parents ayant un TPL : l'impact du trouble mental du parent sur l'enfant et le défi de trouver la façon de parler à l'enfant du TPL de son parent.

L'impact sur l'enfant. Du point de vue des intervenants, il s'avère très difficile de démontrer les effets importants du TPL parental sur les enfants. Selon eux, les répercussions sont subtiles et cumulatives.

Si le parent est flamboyant psychotique, on voit clairement les impacts importants sur l'enfant. Mais quand il y a des petites ratés chroniques, comme dans les troubles de la personnalité, l'impact sur l'enfant est plus subtil, plus fin, et c'est plus difficile de démontrer quoi que ce soit.

Par exemple, les enfants de parents ayant un TPL vivent beaucoup d'anxiété et de craintes. Ils se sentent parfois envahis par leurs émotions et se perçoivent comme "méchants ». Certains enfants externalisent leur détresse et d'autres vivent leur désarroi à l'intérieur. Aux dires des intervenants, plusieurs de ces enfants seraient carencés et désorganisés.

Ces enfants sont à la dérive. La mère n'en prend pas soin et ne permet pas le placement.

... Dans quelle mesure pouvons-nous laisser des enfants vivre au quotidien avec un parent qui est émotionnellement instable de façon chronique? Leurs enfants sont incapables de développer leur confiance aux autres, ils n'ont aucun point de repère. Ils vivent dans un état de stress constant, car il n'y a aucune régularité dans les soins et dans les humeurs. Chaque jour, ils se demandent comment sera leur mère à leur retour de l'école. 
De l'avis des intervenants consultés, certains enfants prennent sur eux l'état de santé de leurs parents et finissent par les prendre en charge. Les intervenants rapportent des histoires qui se répètent sur trois générations. Selon eux, ces enfants ne progressent pas ou peu, et les intervenants déplorent qu'ils n'aient pas de projet de vie ${ }^{4}$. Par ailleurs, lorsque les enfants sont placés, les intervenants subissent de nombreuses attaques de la part des parents et les enfants semblent vivre d'importants conflits de loyauté. S'ils sont maintenus dans cet état durant plusieurs années, les répercussions se font particulièrement sentir à l'adolescence. Ils développent les mêmes symptômes que leurs parents : crises, consommation, impulsivité, autodestruction, et la même façon pathologique d'être en relation. À ce stade du développement, l'enjeu semble être différent parce qu'ils ne sont plus uniquement victimes du parent; ils deviennent acteurs.

\section{La jeune se bat pour ne pas être comme sa maman. Mais si on ne lui dit pas qu'elle a des tendances à être comme sa maman, elle n'apprendra pas à se connaître, elle n'a pas de repères, rien. Éventuellement, c'est sûr qu'elle va reproduire les comportements de sa maman.}

Les intervenants vivent aussi une certaine impuissance à protéger les enfants des comportements dysfonctionnels épisodiques et imprévisibles de leurs parents. Selon eux, étant donné que la situation peut changer sans préavis, l'évaluation des risques actuels et futurs est difficile et leur gestion susciterait beaucoup d'anxiété.

Comment expliquer à l'enfant. Nombreux sont les intervenants qui notent leur difficulté à parler à l'enfant du trouble mental de son parent. Si certains se disent mal à l'aise, tous se considèrent mal équipés pour le faire. Quelques-uns sont réticents à aborder le sujet car ils veulent protéger l'enfant et préserver l'idéalisation du parent. Ils veulent aussi éviter que le jeune se sente responsable de son parent. Les intervenants notent que pour bien traduire à un enfant ce que vit son parent, pour trouver les mots justes pour le dire, il faut qu'eux-mêmes comprennent très bien le problème ce qui n'est pas souvent le cas. Certains intervenants préfèrent de pas aborder le sujet du trouble mental du parent avec les adolescents car ceux-ci se questionnent sur leur propre santé mentale et les intervenants craignent qu'une telle conversation ne provoque de l'anxiété chez le jeune.

Quand le parent l'accepte (son diagnostic), on peut plus facilement aborder le sujet avec le jeune qui a peur d'aborder ces sujets-là. Ils ne veulent pas étiqueter leur parent, car ils ont peur d'être comme eux. Quand le parent est suivi, c'est moins menaçant pour l'enfant. Quand le diagnostic est vague, c'est beaucoup plus difficile.

4. Un projet de vie est une situation dans laquelle l'enfant est maintenu dans un milieu stable et permanent, favorisant le développement d'un lien d'attachement sécurisant. Cette situation comporte deux dimensions: une dimension physique, c'est-à-dire un milieu de vie et une dimension affective, à savoir une personne significative avec qui l'enfant vit et peut développer un lien d'attachement (CJM-IU, Site Internet). 


\section{Les difficultés d'intervention liées au contexte environnemental}

Les difficultés liées à l'organisation interne des services en centre jeunesse. Selon les intervenants rencontrés, les conditions mêmes de leur pratique en centres jeunesse augmentent la difficulté de travailler auprès de familles touchées par un TPL. Ainsi, en raison du nombre élevé de familles dont ils ont la charge, les intervenants n'ont pas le temps de gérer les nombreuses demandes de ces parents.

Je trouve que c'est une charge lourde d'avoir ces dossiers. Le nombre de visites est plus fréquent afin de valider la sécurité de l'enfant. J'ai 4 dossiers de ce type qui demandent beaucoup d'interventions (téléphones fréquents, visites surprises, etc.).

Les situations sont trop pressantes. 5 enfants, 5 conjoints différents. Les interventions sont toujours mises en échec. Je n'ai pas de prise. Eux ils ne bougent pas. Ils ne sont jamais satisfaits. Ils utilisent les services quand ça fait leur affaire après, ce n'est plus bon.

Des intervenants soulignent également des problèmes reliés au roulement du personnel et au statut d'emploi de certains intervenants. Par exemple, les intervenants à temps partiel occasionnel ou sur la liste de rappel connaissent peu la clientèle et n'ont pas le temps ou la possibilité de développer une relation de confiance avec ces parents. Plusieurs d'entre eux se sentent isolés et laissés à eux-mêmes et se disent peu portés à faire appel à l'équipe des consultants ${ }^{5}$ pour obtenir de l'assistance et du soutien. Beaucoup d'intervenants déplorent le manque de concertation concernant l'intervention auprès de ces familles ainsi que l'absence d'une philosophie d'intervention commune aux services psychosociaux et de réadaptation.

Les difficultés liées à la collaboration avec les partenaires externes. II existe certaines difficultés avec les partenaires externes, notamment avec le milieu judiciaire et celui de la psychiatrie adulte.

Le travail au tribunal. Selon les propos des intervenants, il est très difficile de présenter un dossier au tribunal et de le défendre lorsque le parent présente un TPL. Ils soulignent que ces parents ont de très bonnes habiletés devant les juges et arrivent à donner une "bonne performance » en se présentant de façon relativement adéquate. D'après eux, la dynamique de ce trouble fait en sorte que les parents peuvent se montrer " séducteurs " créant ainsi de la confusion et de la division.

5. Cette équipe est formée de professionnels (psychologues et psycho éducateurs) qui agissent à titre de consultants auprès des intervenants et des équipes du centre jeunesse. Leur rôle est d'apporter un éclairage complémentaire aux intervenants afin qu'ils aient une meilleure compréhension de la situation pour ajuster au besoin leurs interventions. 
Certains intervenants font également état de leur manque de crédibilité Iorsqu'ils se présentent devant le tribunal et admettent avoir de la difficulté à faire face aux préjugés envers les services de protection de la jeunesse, notamment lorsque le parent souffre d'un TPL.

Et il y a tellement de tabous, l'image des services sociaux est tellement ternie (...) que lorsque les intervenants se retrouvent au tribunal pour parler des troubles de comportement d'un enfant et faire-valoir que le parent a un trouble de santé mentale et qu'il est TPL, c'est nous qui nous faisons ramasser à coup de poing dans le front, et par les avocats, et par le juge.

De plus, selon l'impression des répondants rencontrés, l'expertise médicale et psychologique serait régulièrement privilégiée à celle des travailleurs sociaux et des éducateurs, au détriment selon eux, des besoins des enfants. Ils soulignent devoir souvent faire plusieurs requêtes avant de convaincre le tribunal des impacts nocifs sur l'enfant. II semble qu'il soit rare qu'une ordonnance oblige un parent à subir une évaluation psychiatrique dans le cas d'un parent ayant un TPL.

En Cour, notre impression n'est pas suffisante même si on travaille sur ce dossier depuis des années. II faut le prouver par des faits et ce n'est pas évident. Par contre, le psychiatre voit la mère 45 minutes, déclare que la mère est apte à reprendre son enfant et cela passe au tribunal. C'est dangereux.

La collaboration avec la psychiatrie adulte. Les participants notent combien il est difficile d'obtenir un diagnostic clair et précis et souhaitent que leur organisation, le CJM-IU, interpelle ses partenaires du domaine de la santé mentale en vue d'instaurer des consultations multidisciplinaires pour les parents.

La consultation multidisciplinaire pourrait donner des résultats positifs: un regard "multi » porté sur la situation, le partage d'informations et d'hypothèses de compréhension, le partage de travail [...]. On est appuyé et validé et, comme le cas est connu, la continuité des services s'ensuit.

En même temps, du point de vue des intervenants, ils soulignent la présence d'importants problèmes quand les parents reçoivent des services en psychiatrie adulte. D’après eux, les psychiatres ne tiennent pas toujours compte de la situation de l'enfant dans l'évaluation du patient. Certains intervenants souhaitent que l'expert en santé mentale puisse évaluer les capacités du parent à prendre soin de son enfant plutôt que de poser un diagnostic. Ils soulignent également combien la lecture de la situation par les psychiatres est différente de celle des intervenants psychosociaux.

On est confronté au fait que des fois les psychiatres vont dire au parent: "Ben oui, t'es apte à le reprendre ton enfant, tu prends ta médication, depuis tant de temps, tu es stable ", mais ils ne 
tiennent pas compte des difficultés que l'enfant a développées et de ses besoins.

Selon d'autres intervenants, les rapports sont difficiles avec certains psychiatres qui sont décrits comme peu accessibles et qui présentent des préjugés envers les intervenants psychosociaux du milieu de la protection de la jeunesse. La transmission d'informations devient dès lors difficile, les règles de confidentialité pouvant faire obstacle à la collaboration.

Moi, j'ai eu un dossier très psychiatrisé avec la mère et ses enfants. Malgré l'autorisation de la mère, son psychiatre refusait de me parler, de me donner des informations, de me fournir des rapports. II disait qu'il n'avait pas à s'adresser à moi, comme si j'étais une moins que rien et qu'il n'avait pas à se justifier... Donc, il faut que je me fie aux paroles de ma cliente, qui a un trouble de la personnalité, pour essayer d'intervenir auprès d'elle, voir si elle prend sa médication adéquatement, mais je ne sais pas ce qu'il lui a prescrit. Je l'ai appelé, il n'a jamais retourné les appels.

\section{L'impact sur les intervenants}

Au cours des entrevues, les intervenants ont abondamment parlé des fortes émotions que suscitent chez eux les parents ayant un TPL, mais aussi de la façon dont leur institution ne reconnait pas assez la lourdeur de ces dossiers et les difficultés qu'ils éprouvent avec cette clientèle.

Les intervenants vivent de fortes émotions. Les intervenants rapportent ressentir beaucoup de colère, d'impuissance, d'incompétence, d'épuisement et de découragement lorsqu'ils travaillent auprès de parents ayant un TPL; "On est tous sur le bord du burnout ».

Le dénigrement, la violence, les discours négatifs de ces parents peuvent faire en sorte que l'intervenant est fortement miné dans son estime de soi, et ce, particulièrement chez les plus jeunes en début de pratique, qui ont peu d'expérience. Il y a une usure avec ces dossiers. L'intervenant est constamment dans l'affrontement.

Selon certains intervenants, ces sentiments négatifs peuvent susciter le désinvestissement affectif, parfois même l'abandon du travail avec ce parent. II peut devenir difficile de bien accueillir les parents ayant un TPL, d'être empathique à leurs difficultés sans intervenir de façon réactive. Ils notent parfois leur envie de les éviter, de les laisser tomber ou de placer les enfants rapidement. De l'avis des participants, certaines réactions d'intolérance et d'excès de prudence ou d'acharnement dans l'intervention, telles vouloir davantage que ce que le client est prêt à faire, peuvent aussi se manifester. Selon eux, dans ces situations, les interventions deviennent moins bien adaptées et les décisions risquent de ne pas toujours être prises de façon objective. 
Est-ce un coup d'épée dans l'eau [de travailler avec ces parents]? Serions-nous mieux d'investir ailleurs? Jusqu'où devons-nous persévérer? C'est grugeant. C'est lourd à porter.

Selon les participants, les parents présentant un TPL les désorganisent et les déstabilisent. Ces derniers semblent bien savoir comment fonctionnent les systèmes de plaintes et s'en serviraient régulièrement. Un intervenant rapporte qu'il a dû composer avec une enquête sur sa pratique en raison d'une plainte reliée à une intervention clinique, qu'il disait pourtant guidée par des données probantes. Un chef de service explique :

Toutes les plaintes que je reçois, que ce soit du comité des usagers ou du commissaire aux plaintes, ce n'est que de parents TPL. Je n'ai que rarement eu des plaintes fondées. Cela crée énormément de litiges entre les organismes, entre les gestionnaires, parce qu'on ne comprend pas, parce que le parent fait du clivage...

Les personnes rencontrées ont également exprimé le besoin de mieux comprendre l'impact que ces parents exercent sur eux. Certains intervenants ont souligné qu'ils aimeraient pouvoir changer leurs attitudes, leurs conceptions et leurs craintes à l'égard du TPL. Ils expriment le besoin qu'une période d'échange soit formellement mise en place avec leur consultant clinique ou leur équipe pour leur permettre de partager ce qu'ils vivent par rapport à ces familles, de mieux comprendre les enjeux relationnels ainsi que la dynamique de ces parents.

Manque de reconnaissance de l'établissement. Selon les participants, les dossiers de parents TPL mobilisent une grande quantité de leur énergie et demandent une somme de travail beaucoup plus grande que les autres dossiers.

Les objectifs doivent être rencontrés en tant de temps. Mais quand il y a un trouble mental chez le parent, tu te fais dire que t'es mal organisé... On nous demande d'être spécialistes en tout. C'est trop. On a des habilités dans notre pratique, mais pas toutes.

Comme chef de service, je suis confronté à ce que j'appelle souvent "les dossiers tue monde ". On passe des heures à reprendre, à réécrire, à envoyer des courriels, à repasser au commissaire des plaintes....Tout ceci est fait par un seul soldat sur la ligne de front, pendant que 350 généraux derrière lui disent quoi faire.

Les intervenants ne se sentent pas toujours soutenus par leur organisation, face aux grandes difficultés, voire la détresse qu'ils vivent face à cette clientèle. Ils estiment que la surcharge de travail causée par ces parents ou encore l'isolement et l'épuisement que ces situations entraînent n'est pas suffisamment reconnues.

Je dirais que dans $50 \%$ de mes dossiers, il y a une problématique de santé mentale. Chaque jour, je me dis « je vais devenir folle! » Est-ce qu'on prend en considération aussi la gravité de l'effet 
que ça nous fait à nous? Parce que moi je suis envahie par $25 \%$ à $35 \%$ de mon "case load". Je n'ai pas de poigne dans ces dossiers-là. Je vis un sentiment d'impuissance au quotidien. À un moment donné, t'es pu capable. Pis t'as rien pour t'accrocher.

Ils souhaitent que leur établissement se préoccupe des difficultés qu'ils vivent face à cette clientèle et désirent que leurs préoccupations soient validées et que l'importance de cette problématique au sein des familles dont ils ont la charge soit reconnue. Les intervenants considèrent comme une nécessité que des mesures soient prises rapidement pour faciliter leur travail.

\section{Discussion}

L'objectif général de cette étude exploratoire qualitative était de rapporter les constatations, préoccupations et difficultés des intervenants du milieu de la protection de la jeunesse qui travaillent auprès de familles touchées par un TPL parental. Les intervenants soulignent qu'ils se trouvent au centre d'un ensemble de contingences qui rend leur intervention particulièrement complexe et exigeante (voir graphique 1). Non seulement doivent-ils faire face aux besoins et à la souffrance des enfants de parents ayant un TPL, mais ils doivent également composer avec les demandes, les besoins et la détresse de leurs parents sans toujours avoir les outils pour le faire. Ils rapportent aussi être confrontés aux exigences de leur établissement qui, d'après eux, ne sont pas adaptées à la complexité et à la sévérité des problèmes associés à ce trouble mental. Enfin, ils estiment que dans ces dossiers, il s'avère particulièrement difficile de transiger avec l'autorité judiciaire ou de collaborer avec les professionnels qui œuvrent dans le domaine de la santé mentale adulte. Du point de vue des intervenants, tenir compte de l'ensemble de ces éléments représente un défi important qu'ils doivent relever dans leur pratique quotidienne et qui les affecte sur le plan personnel.

Les difficultés d'intervention liées au contexte interpersonnel. Les difficultés rencontrées par les intervenants mettent en lumière d'une part leur sentiment de méconnaitre les manières d'être et de faire avec cette clientèle mais également leur souffrance face aux aléas et aux impasses inhérents à relation d'aide avec les personnes souffrant d'un TPL (Bessette, 2010). Or, il est reconnu dans la littérature que situer le TPL dans un cadre théorique et conceptuel est essentiel pour "comprendre avant d'intervenir » (Bergman \& Eckerdal, 2000; Cousineau, 1996). Ces connaissances permettent de développer l'empathie et la sensibilité nécessaire à une intervention efficace et de qualité auprès de ces parents (Bouchard, 2010; Laporte, 2007) et de changer les attitudes envers cette clientèle (Bodner, Cohen-Fridel, \& lancu, 2011).

Comme ceux de tout autre milieu et profession, les intervenants qui travaillent en protection de la jeunesse, sont particulièrement interpellés par la clientèle ayant un TPL. Cependant, et il faut le souligner, ces derniers doivent en plus agir dans le cadre d'une loi d'exception, où l'intervention est effectuée sous contrainte et dont la finalité est déterminée par la loi ou le tribunal (LeBlanc, 2010). Ainsi, à la complexité d'une intervention en contexte d'autorité en protection de la jeunesse, s'ajoutent des conditions de travail difficiles auprès de parents souvent 


\section{Organisation interne}

Difficultés identifiées :

- Organisation des services et conditions de pratique

- Manque de formation sur troubles mentaux et sur la médication

- Lacunes dans la gestion de l'information sur la santé mentale

- Manque de concertation et de cohérence au sein des équipes

- Manque de reconnaissance des

difficultés associées à ce trouble

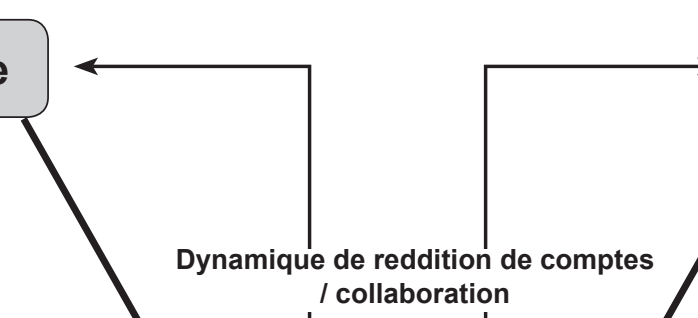

Partenaires externes psychosocial

Difficultés identifiées :

- Absence de diagnostic clair

- Intervention sous contrainte

- Parents qui suscitent des émotions intenses

- « Double » mandat : aide aux parents et protection des enfants

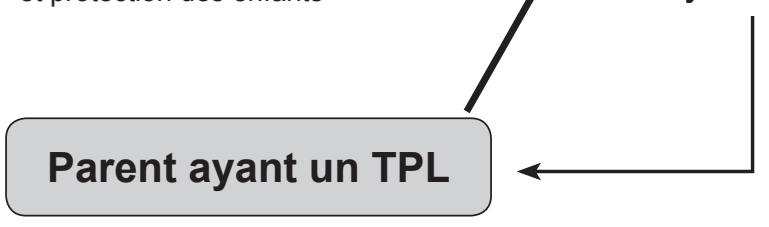

/ collaboration

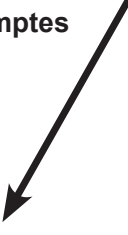

Difficultés identifiées :

- Collaboration insuffisante avec la psychiatrie adulte

- Exigences de la loi sur la protection de la jeunesse

- Présentation des dossiers au tribunal - démonstration de la preuve

- Manque de crédibilité et aux préjugés face à la DPJ

\section{Figure 1. L'intervenant psychosocial : au centre d'une dynamique complexe}


hostiles, hyper vigilants à l'injustice, non-coopérants (Kupper, 2007; Tomison, 2000), vivant dans des conditions psychosociales souvent extrêmement défavorables. $\mathrm{Ce}$ contexte particulier doit être pris en compte en prenant d'abord un soin particulier pour diminuer la détresse du parent avant même de discuter des modalités entourant l'orientation. Dans un texte sur la mobilisation des parents, LeBlanc (2010) suggère que parfois le grand souci des intervenants psychosociaux de protéger les enfants abusés, négligés et souffrants pourrait engendrer une charge émotive puissante qui contribue au désir de précipiter ou de forcer un changement de comportements chez le parent au détriment de leur autodétermination. Ce type de mécanisme peut être particulièrement présent dans le travail auprès de familles touchées par un TPL.

L'absence d'un diagnostic clair chez des parents soupçonnés d'avoir un TPL, identifié par les intervenants comme un enjeu supplémentaire à leur intervention, est une arme à deux tranchants. Bien qu'il existe des bénéfices certains à détecter rapidement les parents souffrant d'un TPL, Fonagy et Bateman (2006) recommandent de prendre aussi en compte les risques d'une stigmatisation. L'impact négatif d'une étiquette de TPL peut être encore plus important en protection de la jeunesse dans le contexte d'une évaluation des habiletés parentales ou de celui d'une décision sur la garde de l'enfant. Ainsi, certains parents pourraient ne pas vouloir consulter ou encore refuser que leur psychiatre partage de l'information avec la protection de la jeunesse de peur d'être stigmatisés par les intervenants. Selon Darlington, Feeney et Rixon (2005b), l'attitude de ces parents est considérée comme un défi important dans l'intervention et semble être un élément majeur dans les décisions portant sur l'évaluation d'un risque immédiat ou futur pour l'enfant. Ainsi, ce qui peut être considéré comme de la résistance ou comme une absence de motivation permettrait aux intervenants ou à l'institution de se dégager de la responsabilité de l'échec d'une intervention (LeBlanc, 2010). Cette auteure suggère par ailleurs que les efforts des intervenants pour mobiliser et responsabiliser les parents, soit de les amener à reconnaître les faits imputés et leurs responsabilités pour qu'ils appliquent des solutions, peuvent souvent être vécus par ces derniers comme « de la mise en accusation » et les démobiliser. Aussi, il se peut que le déni ou la discrétion de certains parents à l'égard de leur problématique mentale et leur méfiance à l'égard des professionnels puissent être une façon adaptée de survivre, influencée par leurs expériences antérieures négatives bien réelles.

II faut souligner par ailleurs que la présence d'un diagnostic clair n'est pas une panacée (Bessette, 2010). Les difficultés que les intervenants éprouvent avec l'impulsivité, l'agressivité, le déni du problème, la méfiance et la dépréciation de la part des parents peuvent se manifester en l'absence d'un diagnostic de TPL chez celui-ci. Bessette (2010) suggère que si les intervenants comprenaient « la dynamique pathologique en jeu, la dynamique relationnelle ainsi que les défenses qui peuvent agir à l'encontre du développement d'une alliance de travail » (p.24), ceux-ci pourraient mieux intervenir auprès de tout parent qui démontre ces types de difficultés, peu importe le type ou la présence d'un diagnostic.

Par ailleurs, l'inquiétude des intervenants quant au risque que les enfants de ces parents développent à leur tour des troubles mentaux est appuyée par plusieurs études. Ainsi, les adolescents de mères ayant un TPL semblent plus 


\section{0}

à risque de développer un trouble de la personnalité, des problèmes d'abus de substance (Cohen, Chen, Crawford, Brook, \& Gordon, 2007), de dépression (Daley et al., 1999) et de démontrer des comportements violents qui persistent jusqu'à l'âge adulte (Johnson et al., 2000; Cohen, Crawford, Johnson, \& Kasen, 2005).

Les difficultés d'intervention liées au contexte environnemental. La littérature souligne que les contraintes et la rigidité de la structure même des services en protection de la jeunesse et les conditions de pratique des intervenants peuvent contribuer à créer des situations de confusion et d'incohérence (Cailhol et al., 2010; Lajoie \& Gauthier, 2006) et ceci serait particulièrement vrai avec des parents présentant un TPL. En effet, la grande mobilité du personnel, la discontinuité de l'intervention et des services, les suivis cliniques irréguliers et parfois discontinus, les ratios de clients élevés, les réorganisations fréquentes et ou encore les contraintes de temps et le manque de ressources peuvent contribuer à augmenter la réactivité des parents et la détérioration de leur condition. St-Jacques et ses collègues (2000) ajoutent également à cette liste le peu de discussion clinique et une certaine rigidité des normes et des procédures d'intervention, que ces auteurs décrivent comme des obstacles organisationnels aux pratiques d'engagement parental dans l'intervention en protection de la jeunesse. D'autres auteurs ont suggéré que certains des comportements dysfonctionnels des personnes ayant un TPL reflètent les failles dans la formation des professionnels qui s'en occupent ou les lacunes dans les services proposés par l'établissement qui les accueille (Dawson, 1988; Fonagy \& Bateman, 2006). Tous ces facteurs structurels et organisationnels peuvent contribuer à diluer les services pour répondre aux besoins de ces familles et à renforcer leur sentiment d'invalidation (Stroud \& Parsons, 2012).

Les répondants soulignent le manque de concertation autant à l'interne qu'avec les différents partenaires. Or, dans ces dossiers, la concertation et le travail d'équipe sont essentiels pour accéder à des expertises multidisciplinaires, partager la lecture des situations et éviter le clivage, harmoniser les pratiques et favoriser la nécessaire cohérence. Ceci aiderait également à réduire l'anxiété des intervenants et à mieux aider les familles (Cailhol et al., 2010; Darlington, Feeney \& Rixon, 2005a; Hetherington, Baistow, Katz, Mesie, \& Trowell, 2002). L'absence de collaboration entre les services de protection de l'enfance et les services de santé mentale adulte soulevée par les intervenants semble reposer sur l'absence de structures et de politiques qui appuient et encouragent cette collaboration (Byrne, Hearle, Plant, Barkla, Jenner, \& McGrath, 2000), le manque de ressources pour établir et maintenir ces contacts (Darlington et al., 2005a; Hetherington et al., 2002) et sur l'existence de différentes politiques de confidentialité et de préjugés de part et d'autre (Darlington et al., 2005a). Dans un article sur cette question, Caihol et ses collègues (2010) plaident pour que les professionnels de la communauté et ceux qui travaillent en santé mentale puissent « unir leurs forces et mettre de côté leurs différends afin d'améliorer la cohérence des soins publics offerts aux personnes présentant un trouble sévère de la personnalité » (p.437).

Les intervenants soulignent également la difficulté de démontrer au tribunal de la jeunesse l'impact négatif que peut avoir un parent ayant un TPL sur son enfant. La littérature confirme que les professionnels du milieu judiciaire ont très peu de connaissances sur ce trouble de santé mentale (Kupper, 2007) et sur 
les enjeux pour le parent ayant un TPL lorsqu'il doit se présenter à la cour dans les cas de protection de la jeunesse. Ces mêmes auteurs soulignent d'ailleurs la nécessité de former la magistrature sur ce trouble mental.

La détresse des intervenants. Les répondants affirment vivre de la détresse dans leur travail auprès de cette clientèle. Tous les guides de meilleures pratiques dans le domaine du TPL soulignent la nécessité qu'une forme de supervision clinique et de soutien en groupe soit en place pour aider les membres de l'équipe (Linehan, 1993; National Health and Medical Research Council, 2012; NHS, 2009). Pour intervenir adéquatement auprès de cette clientèle, les intervenants doivent pouvoir exprimer et normaliser ce qu'ils vivent, valider leurs expériences et prendre le recul nécessaire pour comprendre et mieux gérer les impasses cliniques. Ils doivent être supervisés afin que leur leurs réactions émotives à l'égard de cette clientèle ne se traduisent pas par des interventions contre thérapeutiques (Cousineau, 1996; Normandin \& Ensink, 2007). Ainsi les impasses vécues dans le travail avec les clients ayant un TPL ne sont pas toujours l'affaire du client (Bessette, 2010). Selon cette auteure, "l'impasse peut être relationnelle et/ou découler d'un manque de formation adéquate et elle implique habituellement la régulation du contre-transfert» (Bessette, 2010, p. 88). Si l'intervenant peut distinguer sa propre expérience interne de la réalité extérieure de son client (Masterson \& Klein, 1989), s'il peut reconnaître ses sentiments contretransférentiels et les gérer (Bessette, 2010), il pourra être plus empathique aux difficultés du parent et mieux être en mesure d'intervenir adéquatement auprès de tous les membres de la famille. La majorité des intervenants en protection de la jeunesse, qui proviennent des domaines de la psycho éducation et du travail social, ne sont pas formés à explorer leurs sentiments contre-transférentiels (Bessette, 2010) et ne reçoivent pas toujours le support émotionnel qui est suggéré dans le travail auprès de parents ou d'adolescents ayant un TPL. Bien que des services de soutien et de consultation soient en place dans plusieurs centres jeunesse, ceux-ci semblent être surtout utilisés en urgence, à la pièce ou lors de crises (Baillargeon, 1999). Par ailleurs, il ne semble pas exister de tradition de supervision en groupe sur une base régulière.

\section{Forces et faiblesse}

Cette étude comporte certaines limites qu'il est important de considérer. II se pourrait que certains parents identifiés par les intervenants comme ayant un TPL n'aient pas ce diagnostic; il est impossible de le savoir. Bien que l'on puisse penser que des intervenants attribuent de façon excessive ou trop souvent le diagnostic de TPL aux parents difficiles ou non coopérants, deux recherches récentes suggèrent que le nombre de parents ayant un TPL est élevé (24\% des parents, Gaumont, 2010; $22.5 \%$ des mères, Laporte \& Friedland, 2013) et que les intervenants sousestiment plutôt la présence d'un TPL, les intervenants ayant identifié la présence de ce trouble dans $45 \%$ des cas de TPL d'un sous-échantillon de validation.

La cueillette de donnés réalisée dans le cadre d'une entrevue de groupe présente aussi certaines limites. En effet, ce contexte peut amener certaines personnes à s'exprimer moins que d'autres. Bien que les animateurs aient invité tous les participants à prendre la parole et que le contexte d'une réunion d'équipe 


\section{2}

devrait favoriser la participation de tous, il se peut que certains n'aient pas voulu ou pu s'exprimer et que l'apport de chacun aux discussions n'ait pas été équitable. II se peut également que la présence des chefs de service ait inhibé certains participants ou limité l'élaboration de certains thèmes. De plus, les expressions non verbales des participants durant les entrevues n'ont pas été prises en compte. Enfin, l'étude aurait pu bénéficier d'un audit des résultats auprès des participants.

En contrepartie, l'utilisation de la méthode du groupe cible présentait plusieurs avantages. D'abord, les rencontres ont permis de recueillir les données auprès d'un grand nombre d'intervenants. De plus, la synergie du groupe a permis de stimuler la discussion, d'aider les participants à se rappeler collectivement de certains éléments qui auraient pu être oubliés, mais aussi d'enrichir et de nuancer leurs réponses. Finalement cette étude aborde un sujet d'une grande pertinence sur le plan clinique, juridique et organisationnel, mais rarement traité dans la littérature spécialisée.

\section{Conclusion}

Cette étude a permis de dégager les enjeux d'ordre, organisationnel, personnel et clinique propres au contexte de l'intervention auprès de parents ayant un TPL probable ou avéré en protection de la jeunesse. Elle met en évidence que la prise en charge des familles touchées par ce trouble mental requiert un investissement d'attention et de ressources appréciables, sollicitent énormément les intervenants sur le plan affectif et cognitif et leurs posent des défis cliniques quotidiens.

Bien que les difficultés rapportées par les intervenants en protection de la jeunesse soient vécues individuellement, celles-ci demeurent néanmoins une affaire collective et doivent être abordées d'un point de vue systémique. En conséquence, améliorer l'offre de service aux parents présentant un TPL, rehausser l'expertise des intervenants, clarifier les mandats et développer des mécanismes de liaison soustend en premier lieu des solutions d'ordre organisationnel. Différentes avenues telles la mise en place de modalités de soutien clinique (formation continue, supervision, consultation), de structures organisationnelles facilitantes (co-intervention, approche interdisciplinaire, stratégies souples de gestion, cohésion de tous les intervenants impliqués, charge de cas réduite, etc.) (Bouchard, 2010; Desrosiers, 2013) et de mise en œuvre d'interventions plus efficaces et mieux adaptées aux besoins complexes de ces personnes ont été suggérées pour d'autres contextes d'intervention auprès de personnes présentant un TPL (Cousineau, 1996; Lafleur et al., 2004; Laporte, 2007; Morissette \& Parisien, 1997).

Ces solutions pourraient être appliquées et adaptées au contexte de l'intervention en protection de la jeunesse. Les intervenants bénéficieraient par exemple d'outils pour développer un "savoir-être » et un « savoir-faire » et profiteraient d'une structure de prise en charge favorisant le « pouvoir-faire » au sein de l'établissement.

Cinq années après les rencontres auprès des intervenants du CJM-IU, nous constatons que ce centre jeunesse à donner son appui au développement 
d'interventions, de services et de produits novateurs pour soutenir la pratique des intervenants auprès de parents présentant un TPL. Ainsi, un groupe pour mères ayant un TPL est offert depuis quatre ans (Laporte, Ounis, Laviolette, Cherrier, \& Lavoie, 2013), un groupe pour enfants de mères avec TPL est en développement, des consultations avec la psychiatrie adulte sont en place depuis cinq ans (Laporte et al., 2011) et un livre pour enfants de parents ayant un TPL (Laporte \& Fraser, 2013) a été publié pour aider les intervenants à parler aux enfants du trouble mental de leur parent.

II reste cependant beaucoup à faire pour que cette problématique de santé mentale relativement répandue chez les parents suivis en centre jeunesse, reçoive toute l'attention nécessaire des autorités. En outre, les centres jeunesse doivent multiplier leur efforts pour 1) diffuser les initiatives locales à travers tous le réseau des services de protection de l'enfance, 2) concevoir des protocoles d'intervention auprès de parents présentant un TPL appuyés par un cadre institutionnel et 3 ) développer, maintenir et formaliser des processus de liaisons avec les partenaires de la psychiatrie adulte. Des orientations cliniques intégrées et des prises en charge cohérentes, mises au point en collaboration, pourraient ainsi répondre à ce problème de manière mieux organisée et plus homogène sur l'ensemble du territoire desservi par les services de protection de la jeunesse.

Les institutions qui se préoccupent des difficultés des intervenants et prennent en compte les besoins de formation et de développement qu'ils expriment pour adapter et enrichir leur pratique (formation, supervision continue, soutien) contribuent aussi, et de façon importante, à la qualité des interventions offertes aux familles touchées par un TPL et, indirectement, à la promotion du bien-être psychologique des intervenants. Ainsi, un peu à la manière des poupées gigognes les mesures de soutien et de bienveillance placées autour de l'intervenant engloberont également la famille. «Plus on s'occupe du travailleur, plus on le protège et plus on l'encourage, plus il pourra faire la même chose avec les jeunes (et les familles) dont il a la charge » (Ferguson, 2005, p. 794, traduction libre).

\section{Références}

American Psychiatric Association (2013). Manuel diagnostique et statistique des troubles mentaux (5e édition), Paris, France : Masson.

Aviram, R. B., Brodsky, B. S., \& Stanley, B. (2006). Borderline personality disorder, stigma, and treatment implications. Harvard Review of Psychiatry, 14, 249256.

Baillargeon, L. (1999). Synthèse de la consultation dans le dossier "liens avec la psychiatrie ». Rapport final, CJM-IU, Montréal, Québec.
Barlow, S., Spitzer, C., Grabe, H. J., Kessler, C., \& Freyberger, H. J. (2006). Individual characteristics, familial experience, and psychopathology in children of mothers with BPD. American Academy of Child Adolescent Psychiatry, 45, 965-972.

Baribeau, C. (2010). L'entretien de groupe : considérations théoriques et méthodologiques. Recherches qualitatives, 29, 28-49.

Bateman, A., \& Fonagy, P. (2000). Effectiveness of psychotherapeutic treatment of personality disorders. British Journal of Psychiatry, 177, 138143. 
Bateman, A., \& Fonagy, P. (2006). Mentalization-based treatment for borderline personality disorder: A practical guide. Oxford, On: Toronto University Press.

Bateman, A., \& Fonagy, P. (2009). Randomized controlled trial of outpatient mentalization-based treatment versus structured clinical management for borderline personality disorder. American Journal of Psychiatry, 166, 1355-1364.

Betan, E., Heim, A. K., Conklin, C. Z., Westen, D. (2005). Countertransference phenomena and personality pathology in clinical practice: An empirical investigation. American Journal of Psychiatry, 162, 890-898.

Bergeron-Leclerc, C. (2010). Les soins destinés aux personnes souffrant d'un trouble de personnalité limite: les stratégies gagnantes. Association québécoise des infirmières et infirmiers, Québec, Québec.

Bergman, B., \& Eckerdal, A. (2000). Professionnal skills and frame of work organization in managing BPD. Scandinav Journal of Caring Science, 14, 245-252.

Bessette, M. (2010). Psychothérapie des troubles de la personnalité: quand l'impasse n'est pas du côté du client. Santé mentale au Québec, 35, 87-116.

Biskin, R., \& Paris, J. (2012). Evaluating treatments of borderline personality disorder. Clinical Practice, 9, 425-437.

Bodner, E., Cohen-Fridel, S., \& lancu, I. (2011). Staff attitudes toward patients with borderline personality disorders. Comprehensive Psychiatry, 52, 548555.

Bouchard, S. (2010). Impasses et opportunités dans le traitement des personnes souffrant d'un trouble de la personnalité limite. Santé mentale au Québec, 35, 61-85.

Byrne, L., Hearle, J., Plant, K., Barkla, J., Jenner, L., \& McGrath, J. (2000). Working with parents with a serious mental illness: what do service providers think? Australian Social Work, 53, 21-26.

Cailhol, L., Bouchard, S., Belkadi, A., Benkirane, G., Corduan, G., Dupouy, S., ...Guelfi, J. D. (2010). Acceptabilité et faisabilité de la psychothérapie par les patients avec trouble de personnalité limite. Annales médico-psychologiques, 168, 435-439.

Cleary, M., Siegfried, N., \& Walter, G. (2002). Experience, knowledge and attitudes of mental health staff regarding clients with a borderline personality disorder. International Journal of Mental Health Nursing, 11, 186-191.

Cohen, P., Chen, H., Crawford, T. N., Brook, J. S., \& Gordon, K. (2007). Personality disorders in early adolescence and the development of later substance use disorders in the general population. Drug Alcohol Dependence, 88, 71-84.

Cohen, P., Crawford, T., Johnson, J., \& Kasen, S. (2005). The children in community study of developmental course of PD. Journal of Personality Disorders, 19, 466-486.

Coulborn Faller, K., \& Bellamy, C. (2000). Mental health problems and child maltreatment, National Resource Center on Child Maltreatment, Georgia, USA.

Cousineau, P. (1996). Intervention auprès du patient limite : objectivité et subjectivité. Revue santé mentale au Québec, 22, 1 , 5-15.

Crawford, M., Rutter, D., Price, K., Weaver, T., Josson, P., Tyrer...Taylor, E. (2007). Learning the lessons: a multi-method evaluation of services for people with personality disorders. Report for the National Co-ordinating Centre for NHS Service Delivery and Organisation R\&D (NCCSDO), London.

Daley, S. E., Hammem, C., Burge, D., Davila, J., Lindberg, N., \& Herzberg, D. S. (1999). Depression and axis II in an adolescent community sample: Concurrent and longitudinal associations. Journal of Personality Disorders, 13, 47-59. 
Darlington, Y., Feeney, J. A., \& Rixon, K. (2005a). Interagency collaboration between child protection and mental health services: Practices, attitudes and barriers. Child Abuse \& Neglect, 29, 1085-1098.

Darlington, Y., Feeney, J. A., \& Rixon, K. (2005b). Practice challenges at the intersection of child protection and mental health. Child and Family Social Work, 10, 239-247.

Dawson, D. F. (1988). Treatment of the borderline patient, relationship management. Revue canadienne de psychiatrie, 33, 370-374.

Denis, J. F. (1990). Le problème des troubles de la personnalité en psychiatrie. Revue canadienne de psychiatrie, 35, 208-214.

Desrosiers, L. (2013). Modèle de l'engagement et de l'abandon de traitement de l'adolescent avec trouble de personnalité limite. Thèse de doctorat, Université de Montréal, Montréal, Québec.

Ferguson, H. (2005). Working with violence, the emotions and the psycho-social dimensions of child-protection. Social Work Education, 24, 781-795.

Fonagy, P., \& Bateman, A. (2006). Progress in the treatment of BPD. The British Journal of Psychiatry, 188, 1-3.

Fruzzetti, A. E., Shenk, C., \& Hoffman, P. D. (2005). Family interaction and the development of BPD: A transactional model. Development and Psychology, 17, 1007-1030.

Gaumont, C. (2010). Prévalence des problématiques de santé mentale chez la clientèle du Centre jeunesse de la Montérégie. Centre jeunesse de la Montérégie, Québec, Québec.

Hetherington, R., Baistow, K., Katz, I., Mesie, J., \& Trowell, J. (2002). The welfare of children with mentally ill parents: Learning from inter-country comparisons. New York, NY: John Wiley \& Sons.
Johnson, J. G., Cohen, P., Smiles, E., Kasen, S., Oldham, J. M., Skodol, A. E., \& Brook, J. S. (2000). Adolescent personality disorders associated with violence and criminal behavior during adolescence and early adulthood. American Journal of Psychiatry, 157, 1406-1412.

Kupper, D. (2007). Borderline personality disorder and the attorney-client relationship: managing the difficult legal client to maximize positive outcomes. In Wolff, CEB Topics, Version 21.

Lafleur, I., St-Onge, M., Rouleau, D., Tremblay, G., \& Villeneuve, E. (2004). L'adaptation des services aux personnes ayant un trouble de personnalité limite : une nouvelle compréhension pour la faciliter? Revue intervention, 121, 61-66.

Lajoie, R., \& Gauthier, L. (2006). Des jeunes laissés à l'abandon par une société en quête de cohérence. Mémoire, Commission parlementaire des affaires sociales du Québec.

Laporte, L. (2007). Intervenir auprès de parents ayant un trouble de personnalité limite : un défi de taille pour les centres jeunesse. Revue santé mentale au Québec, 2, 97-114.

Laporte, L. \& Fraser, D. (2013). La maison des intempéries: Vivre avec un parent ayant un trouble de personnalité limite, Québec, Québec : Éditions Midi trente.

Laporte, L., \& Friedland, N. (2013). Les mères ayant un trouble de personnalité limite et le système de protection de la jeunesse : Déterminants et conséquences des disparités entre besoins et services. Journées professionnelles Batshaw, Montréal, Juin 2013.

Laporte, L., Helde, M., Gaudet, N., \& Lachance, O. (2011). Le TPL chez les parents des usagers des services de protection de l'enfance: un défi additionnel pour les intervenants... et des pistes de solutions. Colloque en maltraitance, Hôpital Ste-Justine. 
Laporte, L., Ounis, L., Laviolette, I., Cherrier, J.-F., \& Lavoie, S. (2013). Groupe pour parents ayant un trouble de personnalité limite dont l'enfant est suivi en protection de l'enfance. Dans R. Coutanceau et J. Smith (dir.), Troubles de la personnalité : $\mathrm{Ni}$ psychotiques, ni névrotiques, ni pervers, ni normaux..., (p. 356-376). Paris, France : Éditions Dunod.

LeBlanc, C. (2010). La mobilisation au changement dans un contexte d'intervention sous contrainte en protection de la jeunesse : enjeux, pièges et outils. CJM-IU, Montréal, Québec.

Linehan, M. M. (1993). Cognitive-behavioral treatment of borderline personality disorder. New York, NY: Guilford Press.

Linehan, M. M., Comtois, K. A., Murray, A. M., Brown, M. Z., Gallop, R. J., Heard, H. L., . . Lindenboim, N. (2006). Two-year randomized controlled trial and followup of dialectical behavior therapy vs therapy by experts for suicidal behaviors and borderline personality disorder. Archives of General Psychiatry, 63, 757766.

Marziali, E., Damianakis, T., \& Trocmé, N. (2003). Nature and consequences of personality problems in maltreating caregivers. Families in Society, 84, 530538.

Masterson, J. F., \& Klein, R. (1989). Psychotherapy of the disorders of the self. The Masterson Approach. New York, NY: Brunner/Mazel.

Mayer, R., \& Deslauriers, J. P. (2000). Quelques éléments d'analyse qualitative: l'analyse de contenu, l'analyse ancrée, l'induction analytique et le récit de vie. Dans R. Mayer, F. Ouellet, M.C. Saint-Jacques, M. C., \& D. Turcotte (dir.) Méthodes de recherche en intervention sociale, (p. 71-90), Montréal, Québec: Gaëtan Morin.

Ministère de la Santé et des Solidarités (2008). Souffrances et troubles psychiques : rôle et place du travailleur social. Direction de la Santé et de l'Action sociale, France.
Morissette, L., \& Parisien, M. (1997). Contribution de l'hôpital au traitement du patient borderline. Revue santé mentale au Québec, 22, 30-42.

National Health and Medical Research Council (2012). Clinical Practice Guideline for the Management of Borderline Personality Disorder. Melbourne, Australia.

NHS - National Institute for Heath and Clinical Excellence (2009). Borderline personality disorder: treatment and management. NICE clinical guideline 78, Developed by the National Collaborating Centre for Mental Health, London, England.

Normandin, L., \& Ensink, K. (2007). GAC : grille d'analyse du contre-transfert dans le traitement des troubles sévères de la personnalité. Revue santé mentale au Québec, 32, 1.

Palmer, M., Larkin, M., Visser, R., \& Fadden, G. (2010). Developing an interpretative phenomenological approach to focus group data. Qualitative Reseach in Psychology, 7, 99-121.

Paris, J. (1996). Social Factors in the Personality Disorders: A biopsychosocial approach to etiology and treatment. Cambridge, UK, Cambridge University Press.

Paris, J. (2009). The treatment of BPD: Implications of research on diagnosis, etiology and outcome. Annual Review of Clinical Psychology, 5, 75-88.

Paris, J. \& Zweig-Frank, H. (2001). A 27-year follow-up of patients with borderline personality disorder. Comprehensive Psychiatry, 42, 482-487.

Perepletchikova, F., Ansell, E., \& Axelrod, S. (2012). Borderline personality disorder features and history of childhood maltreatment in mothers involved with child protective services. Child Maltreatment, 17, 182-190. 
Ravon, B., Decrop, G., Ion J., Laval, C., \& Vidal-Naquet, P. (2008). Épreuves de professionnalité : repenser l'usure professionnelle des travailleurs sociaux. Rapport pour l'observatoire national de la pauvreté et de l'exclusion sociale (ONPES), 260 pages. Lyon, France.

Rutter, M., \& Quinton, D. (1984). Parental psychiatric disorder: Effects on children. Psychological Medicine, 14, 853-880.

Stanley, N., \& Manthorpe, J. (2001). Reading mental health inquiries: Messages for social work. Journal of Social Work, 1, 77-99.

Stanley, N., Penhale, B., Riordan, D., Barbour, R. S., \& Holden, S. (2003). Child protection and mental health services: Interprofessionnal responses to the need of mothers. Bristol, UK: The Policy Press.
St-Jacques, M. C., Lessard, G., Beaudoin, A., \& Drapeau, S. (2000). Les pratiques d'implication parentale dans l'intervention en protection de la jeunesse. Beauport, Québec : CJQ-IU.

Stroud, J. \& Parsons, R. (2012). Working with borderline personality disorder: A small-scale qualitative investigation into community psychiatric nurses' constructs of borderline personality disorder. Personality and Mental Health, 7, 242-253.

Tomison, A. M. (2000). Evaluating child abuse prevention programs, NCPCH Issues Paper no. 12. Melbourne, Australie: AIFS.

Touré, E. H. (2010). Réflexion épistémologique sur l'usage des focus groups: fondements scientifiques et problèmes de scientificité. Recherches qualitatives, 29, 5-27. 